\title{
Involvement of CXCL14 in osteolytic bone metastasis from lung cancer
}

\author{
SOICHI TAKIGUCHI ${ }^{1}$, NATSUKI KORENAGA ${ }^{2}$, KAZUKO INOUE $^{1}$, ERIKA SUGI $^{3}$, \\ YASUFUMI KATAOKA ${ }^{3}$, KIMIHIKO MATSUSUE ${ }^{4}$, KOUJIRO FUTAGAMI ${ }^{2}$, YIN-JI LI ${ }^{5}$, \\ TOSHIO KUKITA ${ }^{5}$, NORIHIRO TERAMOTO ${ }^{6}$ and HARUO IGUCHI ${ }^{7}$
}

\author{
${ }^{1}$ Clinical Research Institute, National Hospital Organization Kyushu Cancer Center, Fukuoka 811-1395; \\ ${ }^{2}$ Department of Pharmacy, Fukuoka University Hospital, Fukuoka 814-0180; Departments of ${ }^{3}$ Pharmaceutical Care \\ and Health Sciences, and ${ }^{4}$ Hygiene Chemistry, Faculty of Pharmaceutical Sciences, Fukuoka University, \\ Fukuoka 814-0180; ${ }^{5}$ Department of Molecular Cell Biology and Oral Anatomy, Faculty of Dental Science, \\ Kyushu University, Fukuoka 812-8582; ${ }^{6}$ Division of Pathology, and ${ }^{7}$ Clinical Research Institute, \\ National Hospital Organization Shikoku Cancer Center, Matsuyama 791-0280, Japan
}

Received December 2, 2013; Accepted January 23, 2014

DOI: $10.3892 /$ ijo.2014.2293

\begin{abstract}
To investigate the molecular mechanisms of lung cancer-induced bone metastasis, we established a boneseeking subclone (HARA-B4) from a human squamous lung cancer cell line (HARA) using an in vivo selection method. We compared comprehensive gene expression profiles between HARA and HARA-B4, and identified the critical factors for the formation of bone metastasis using in vitro and in vivo assays. The number of bone metastatic colonies in the hind legs was significantly higher in HARA-B4-inoculated mice than in HARA-inoculated mice at 4 weeks after inoculation. In addition, visceral (adrenal) metastases were not found in HARA-B4-inoculated mice at autopsy, suggesting an increase in cancer cell tropism to bone in HARA-B4. Based on a comprehensive gene expression analysis, the expression level of CXC chemokine ligand 14 (CXCL14) was 5-fold greater in HARA-B4 than in HARA. Results of a soft agar colony formation assay showed that anchorage-independent growth ability was 4.5-fold higher with HARA-B4 than with HARA. The murine pre-osteoblast cell line MC3T3-E1 and the preosteoclast/macrophage cell line RAW264.7 migrated faster toward cultured HARA-B4 cells than toward HARA cells in a transwell cell migration assay. Interestingly, CXCL14 was shown to be involved in all events (enhancement of cancer cell tropism to the bone, anchorage-independent growth and/ or recruitment of bone marrow cells) based on siRNA experiments in HARA-B4 cells. Furthermore, in clinical specimens
\end{abstract}

Correspondence to: Dr Haruo Iguchi, Clinical Research Institute, National Hospital Organization Shikoku Cancer Center, Minamiumemotomachi Ko160, Matsuyama, Ehime 791-0280, Japan E-mail: higuchi@shikoku-cc.go.jp

Key words: CXCL14, bone metastasis, lung cancer, anchorageindependent growth, chemotaxis of lung cancer-induced bone metastasis, expression of CXCL14 was observed in the tumor cells infiltrated in bone marrow in all specimens examined. CXCL14 was able to promote bone metastasis through enhancement of cancer cell tropism to the bone and/or recruitment of bone marrow cells around metastatic cancer cells.

\section{Introduction}

Recently, bone metastasis has been increasing in patients with various cancers. This results in bone pain, pathological fractures, neurological deficits, and/or hypercalcemia, which are related to a decrease in the quality of life (QOL) in patients with bone metastasis. Thus, it is important to understand how these patients should be managed.

Cancer cells are distributed hematogenously to the bone (1). The process of such hematogenous spread in soft tissue metastasis includes shedding of cancer cells from a primary tumor into the circulation, colonization in a target organ, extravasation into the surrounding tissue, initiation and maintenance of growth, and vascularization of the metastatic tumor (2). However, in bone metastasis, an additional step in which cancer cells invade and proliferate in the bone matrix is necessary (3-5). Current evidence suggests that osteoclasts play a crucial role in this step during tumor-associated osteolysis, and several osteoclastogenic factors, i.e., parathyroid hormone-like hormone (PTHLH) (6,7), secreted matrix metalloproteases (MMPs) (8), interleukin-6 (IL-6) (9), receptor activator of nuclear factor $\kappa \mathrm{B}$ ligand (RANKL) (10), macrophage inflammatory protein-1 $\alpha$ (MIP-1 $\alpha)(11)$, interleukin-8 (IL-8) $(12,13)$, and granulocyte-macrophage colony-stimulating factor (GM-CSF) $(14,15)$ have been implicated in the activation of osteoclastic bone resorption during tumor-associated osteolysis in solid tumors. These factors, released by metastatic tumor cells in the bone marrow, stimulate differentiation and activation of osteoclasts either directly or through osteoblasts, thereby leading to osteolysis and the release of tumor cell 
growth factors from the bone matrix. Based on this evidence for the molecular mechanism of bone metastasis, bisphosphonates (16) and denosumab (17), which inhibit osteoclastic bone resorption, have been developed for the treatment of bone metastasis. Although these drugs are widely used, their clinical benefit is limited. Therefore, the development of a novel drug for the treatment of bone metastasis is necessary.

It is important to identify factors that are involved in bone metastasis because such factors could be attractive therapeutic targets. The present study describes experimental evidence of the role of CXC chemokine ligand 14 (CXCL14), a member of the CXC chemokine family, in the progression of bone metastasis from lung cancer.

\section{Materials and methods}

Cell culture. HARA cells were obtained from the Health Science Research Resources Bank (Osaka, Japan). HARA and its bone-seeking subclones were cultured in RPMI-1640 medium (Invitrogen, Carlsbad, CA, USA) supplemented with $10 \%$ fetal bovine serum (FBS), $50 \mathrm{U} / \mathrm{ml}$ of penicillin, and $50 \mu \mathrm{g} / \mathrm{ml}$ of streptomycin in a humidified atmosphere under $5 \% \mathrm{CO}_{2}$ at $37^{\circ} \mathrm{C}$. The murine pre-osteoclastic/macrophage cell line RAW264.7 and pre-osteoblastic cell line MC3T3-E1 were cultured in $\alpha$-modified minimum essential medium ( $\alpha$-MEM) supplemented with $10 \% \mathrm{FBS}, 50 \mathrm{U} / \mathrm{ml}$ of penicillin, and $50 \mu \mathrm{g} /$ $\mathrm{ml}$ of streptomycin in a humidified atmosphere under $5 \% \mathrm{CO}_{2}$ at $37^{\circ} \mathrm{C}$.

In vivo selection of a bone-seeking HARA subclone. HARA cells $\left(2 \times 10^{5}\right.$ cells) inoculated into the left ventricle of the heart in 5-week-old male nude mice (Clea Japan Inc, Tokyo, Japan) spread mostly to the bone, muscle, and adrenal glands 4-6 weeks after inoculation, suggesting that batches of HARA cells contain mixed subpopulations with different metastatic potentials. To establish a bone-seeking subclone, HARA cells in bone metastases were isolated by the explant outgrowth technique, grown in culture (HARA-B), and reinoculated into the left ventricle of the heart. HARA-B cells that metastasized to bone were then isolated and grown in culture (HARA-B2). This procedure was repeated 4 times until no metastases were detected macroscopically in the adrenal glands (HARA-B4). All animal studies were carried out according to the protocol approved by the National Hospital Organization Kyushu Cancer Center Animal Ethics Committee (permit no. 23-02), in compliance with the Committee guidelines.

Total RNA preparation and DNA microarray. Total RNA was extracted from HARA and HARA-B4 cells using a Qiagen RNeasy kit (Qiagen, Venlo, The Netherlands) according to the manufacturer's instructions. RNA samples were analyzed using a GeneChip Human Genome U133 Plus 2.0 Array (Affymetrix, Santa Clara, CA, USA). The GeneSpring GX 7.3.1 program (Agilent Technologies, Santa Clara, CA, USA) was used to filter gene expression levels. The results were visualized using a Venn diagram, and the genes known to exhibit expression changes of $\geq 5$-fold with larger raw data values of $>100$ were listed. The microarray data described in this report have been deposited in the NCBI GEO database with the accession no. GSE29367.
Reverse transcription and quantitative real-time polymerase chain reaction $(P C R)$. Reverse transcription was performed using the ThermoScript RT-PCR System (Invitrogen) according to the manufacturer's instructions. Quantitative real-time PCR (qRT-PCR) was performed using the ABI PRISM 7000 Sequence Detection System (Applied Biosystems, Foster City, CA, USA) and SYBR Premix Ex Taq (Takara Bio, Otsu, Shiga, Japan). The PCR conditions were as follows: $95^{\circ} \mathrm{C}(10 \mathrm{sec})$ followed by 40 cycles at $95^{\circ} \mathrm{C}(5 \mathrm{sec})$ and $60^{\circ} \mathrm{C}(31 \mathrm{sec})$. The human gene-specific primers were 5'-CGCTACAGCGAC GTGAAGAA-3' and 5'-TTCCAGGCGTTGTACCACTTG-3' for CXCL14, 5'-CCGTCCGATTTGGGTCTGATG-3' and 5'-TCTTTTTCTTCCCAGGTGTCTTG-3' for PTHLH, 5'-CCGTCGCCCATCATCAAGTT-3' and 5'-CTGTCTGGG GCAGTCCAAAG-3' for MMP2, 5'-AAGAAGTGGAATA AGTGGGCTCT-3' and 5'-GGCCGAATAAGGGTCTGGG-3' for adrenomedullin (ADM), 5'-AGCTCTGTCTGGACCCC AAG-3' and 5'-TGGCAACCCTACAACAGACC-3' for IL-8, and 5'-CATGTACGTTGCTATCCAGGC-3' and 5'-CTCC TTAATGTCACGCACGAT-3' for $\beta$-actin.

RNA interference. Stealth Select RNAi duplexes designed to target CXCL14 (siCXCL14) and negative controls designed not to target any known human gene (siNC) were purchased from Invitrogen. For the silencing assay, we transfected $5 \times 10^{5}$ cells (HARA or HARA-B4) with $15 \mu$ l of stock Stealth Select RNAi duplex $(20 \mu \mathrm{M})$ using Lipofectamine RNAiMAX solution (Invitrogen) in a 100-mm diameter culture dish. We harvested total RNA from transfected cells $48 \mathrm{~h}$ after transfection to perform gene expression profiling.

Anchorage-independent proliferation in an agar matrix. Soft agar experiments were performed using a CytoSelect 96-well cell transformation assay kit following the manufacturer's instructions (Cell Biolabs, San Diego, CA, USA). Briefly, the bottom layer contained $50 \mu \mathrm{l}$ of $1 \mathrm{X}$ CytoSelect agar matrix solution and 10\% FBS in Dulbecco's modified Eagle's medium (DMEM), and the top layer contained $75 \mu \mathrm{l}$ of $1 \mathrm{X}$ CytoSelect agar matrix solution, $10 \%$ FBS in DMEM, and $5 \times 10^{3}$ cells. All assays were performed in 96-well plates. The plates were incubated for 1 week at $37^{\circ} \mathrm{C}$ with $5 \% \mathrm{CO}_{2}$ to allow cell proliferation in soft agar. Photographs were taken using an Olympus phase contrast microscope. Volumes of colonies were calculated according to absorbance values at $570 \mathrm{~nm}$ using the MTT assay after the addition of matrix solubilization solution.

Resorbing activity of osteoclasts. Osteoclasts were formed in the rat whole bone marrow cell culture system as described previously (18). Briefly, bone marrow cells were obtained from the tibia and femur of 4-week-old male Sprague Dawley rats and cultured in 24 -well plates $\left(1 \times 10^{6}\right.$ cells/well) in $\alpha$-MEM containing $15 \%$ FBS in the presence of $10^{-8} \mathrm{M} 1 \alpha, 25(\mathrm{OH})_{2} \mathrm{D}_{3}$ and $10 \%(\mathrm{v} / \mathrm{v})$ htROSCM (19). After 4 days of culture, the cells were detached from the culture plates with $0.05 \%$ trypsin and $0.02 \%$ EDTA. Mature osteoclasts were then cocultured with HARA or HARA-B4 $\left(2 \times 10^{3}\right.$ cells/well) on Osteologic discs (BD, Franklin Lake, NJ, USA). After 3 days of culture, attached cells were removed from the discs using 6\% sodium hypochlorite, and von Kossa staining was performed according to the manufacturer's instructions. Images of pits in each well 
HARA

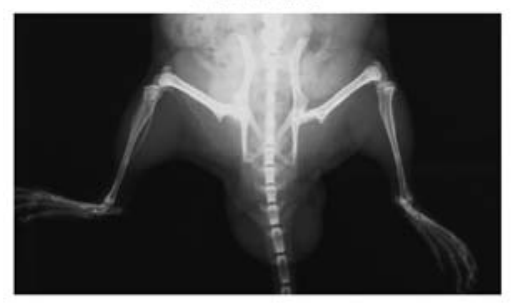

HARA-B4

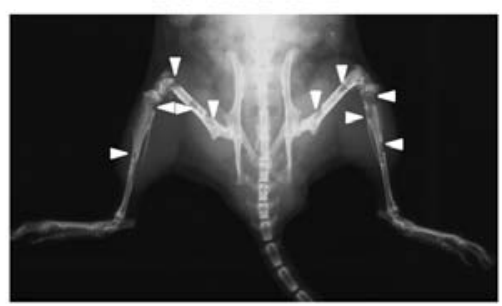

Figure 1. In vivo selection of a bone-seeking HARA subclone. Representative X-ray images showing areas of osteolytic bone destruction (white arrow heads), which were obtained 4 weeks after intracardiac inoculation.

were obtained using inverted microscopy (BZ-8100, Keyence, Japan). The total area of resorption pits and the total number of resorption pits per well were measured using an image analysis system (BZ-II analyzer; Keyence, Japan).

Transwell cell migration assay. Transwell cell migration was evaluated using a 24-well chemotaxis chamber with 3 - and $8-\mu \mathrm{m}$ pore size membranes (BD) for MC3T3-E1 and RAW264.7, respectively. HARA and HARA-B4 $\left(2.5 \times 10^{4}\right.$ cells $\left./ 0.8 \mathrm{ml}\right)$ cells transfected with various RNAi duplexes were cultured in the lower chamber for $16 \mathrm{~h}$, and the medium was then changed to serum-free $\alpha$-MEM $(0.8 \mathrm{ml})$. Concurrently, MC3T3-E1 and RAW264.7 were cultured in serum-free $\alpha$-MEM medium for $12 \mathrm{~h}$ and harvested. Cell suspensions of MC3T3-E1 $\left(1.25 \times 10^{4}\right.$ cells $\left./ 0.5 \mathrm{ml}\right)$ and RAW264.7 $\left(1.25 \times 10^{5}\right.$ cells/0.5 ml) were then added to the upper chamber and incubated for 4.5 and $6 \mathrm{~h}$, respectively, at $37^{\circ} \mathrm{C}$. Non-migrated cells were wiped off with a cotton swab, the filter was stained with Diff-Quik stain solution (Siemens, Munch, Germany), and the remaining cells were counted under the microscope.

Immunohistochemistry. Lung cancer cells (HARA/siNC, HARA-B4/siNC, HARA-B4/siCXCL14) were injected into the left cardiac ventricle of nude mice, which were sacrificed 2 weeks after injection. The legs of the mice were then fixed in buffered formalin, decalcified in 10\% EDTA ( $\mathrm{pH} 7.0$ ), and embedded in paraffin. Human clinical specimens of pulmonary adenocarcinoma bone metastases $(n=7)$, which were obtained by computed tomography-guided needle biopsy, were also subjected to immunohistochemistry for the detection of CXCL14. Sections (5- $\mu \mathrm{m}$ thick) were treated with target retrieval solution (Dako, Glostrup, Denmark) for $20 \mathrm{~min}$ at $95^{\circ} \mathrm{C}$ and subsequently with anti-CXCL14 antibody (1:100, MAB866, R\&D Systems, Minneapolis, MN, USA). CXCL14 was visualized using CSA-II (Dako) and Autostainer Plus (Dako) according to the manufacturer's instructions. Histological sections were also stained with hematoxylin and eosin. All the human specimens for this study were collected after obtaining written informed consent from the patients. This study and the protocols were approved by the Institutional Ethics Committee of National Hospital Organization Shikoku Cancer Center, where the patients were treated.

Statistical analysis. The results are presented as mean \pm SD. Group comparisons were performed using either: i) the nonparametric Mann-Whitney test, or ii) the parametric Student's
Table I. Establishment of a bone-seeking subclone (HARA-B4) from the parental cell line (HARA) that was established from human squamous cell lung cancer.

HARA

HARA-B4

No. of bone metastases

$\begin{array}{lcc}4 \text { weeks } & \mathrm{ND}^{\mathrm{a}} & 15.5 \pm 3.7^{\mathrm{c}} \\ \text { Autopsy } & 26.5 \pm 9.4 & 53.5 \pm 20.6\end{array}$

Incidence of adrenal metastases
Autopsy
$4 / 4$
$4 / 0^{\mathrm{b}}$

The number of bone metastases in the hind legs was counted by radiography at 4 weeks and 7-8 weeks (at autopsy) after inoculation of the cells. The incidence of visceral metastasis (adrenal metastasis) was also examined at autopsy. Mean $\pm \mathrm{SD}(\mathrm{n}=4)$. ${ }^{\mathrm{a}} \mathrm{ND}$, not detected; ${ }^{b} \mathrm{p}<0.05 ;{ }^{c} \mathrm{p}<0.01$ compared to HARA-inoculated mice.

t-test and one-way analysis of variance (ANOVA) followed by Bonferroni's multiple comparison test. Differences were considered significant at $\mathrm{p}<0.05$.

\section{Results}

Establishment of a bone-seeking subclone of HARA and its capacity to metastasize to the bone and adrenal glands. After 4 passages in vivo and in culture, we successfully established a bone-seeking subclone (HARA-B4). We then investigated the metastatic capacity of HARA-B4 compared to the parental line (HARA) in the heart injection model. Radiographs of hind legs were obtained 4 weeks after intracardiac inoculation to detect bone metastatic colonies. Few colonies were detected in HARA-inoculated mice, whereas nearly 15 metastatic colonies were detected in HARA-B4-inoculated mice (Fig. 1 and Table I). The mice were sacrificed at 7-8 weeks post-inoculation when hind leg paralysis was noted or when body weight decreased below $20 \mathrm{~g}$. There were no significant differences in the number of macroscopic bone metastases between HARAand HARA-B4-inoculated mice at autopsy, although it tended to be higher in the HARA-B4-inoculated mice (Table I). All HARA-inoculated mice developed metastases to the adrenal glands in addition to bone, while adrenal metastasis was not found in HARA-B4-inoculated mice (Table I). Metastases to the muscle were also seen in both HARA- and HARA-B4inoculated mice with bone metastasis (data not shown). 

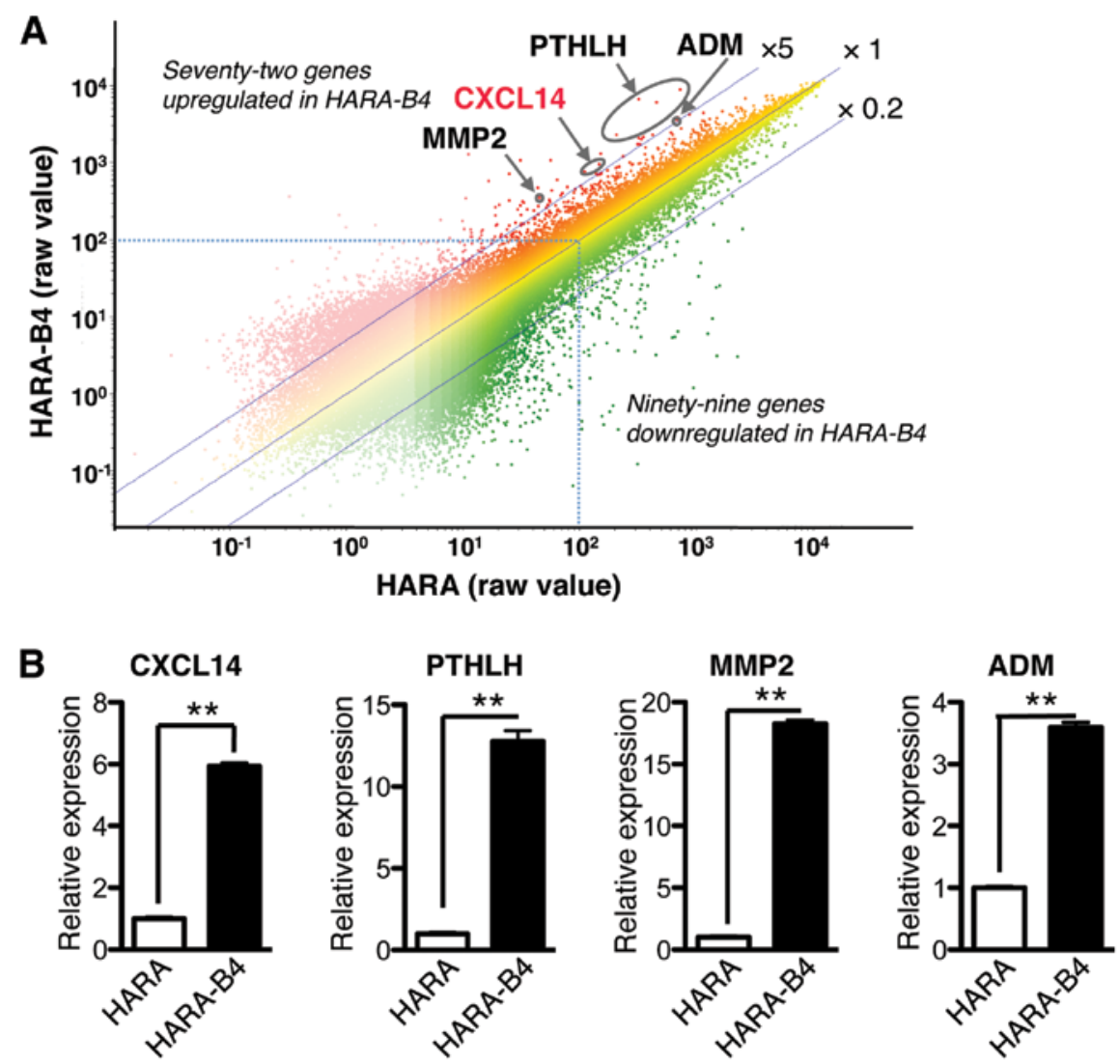

Figure 2. Identification of bone metastasis-related genes. (A) Gene expression arrays were performed for HARA and HARA-B4 cells using Affymetrix GeneChips. In total, 72 genes were upregulated $>5$-fold in HARA-B4 cells compared with HARA cells. Among these, we focused on 4 genes $(P T H L H$, $M M P 2, A D M$ and $C X C L 14$ ) that were likely involved in bone metastasis based on their upregulation in HARA-B4 cells and the fact that they have been previously reported in the context of cancer proliferation and metastasis. (B) To validate the GeneChip data, the expression levels of these genes were assessed by qRT-PCR.

HARA-B4/siNC

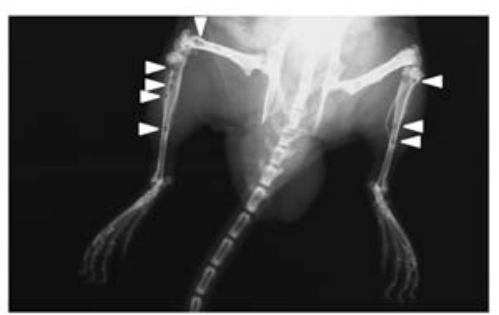

HARA-B4/siCXCL14

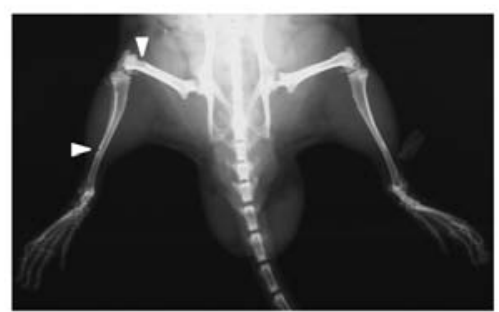

Figure 3. Effect of CXCL14 silencing on bone metastasis in HARA-B4-inoculated mice. Representative X-ray images showing areas of osteolytic bone destruction (white arrow heads), which were obtained 4 weeks after intracardiac inoculation.

Identification of bone metastasis-related genes. In order to elucidate the genes involved in bone metastasis, we compared comprehensive gene expression profiles between HARA and HARA-B4. Gene expression arrays were performed on HARA and HARA-B4 cells using Affymetrix GeneChips. The GeneSpring GX 7.3.1 program (Agilent Technologies) was used to filter gene expression levels. The genes with expression changes of $\geq 5$-fold and raw data values of $>100$ are selected for further analysis. A total of 72 genes were upregulated and 99 genes were downregulated in HARA-B4 compared to HARA (Fig. 2A). To validate the GeneChip data, the expression levels of four differentially expressed genes (PTHLH,
$M M P 2, A D M$ and $C X C L 14$ ) that may participate in metastasis were assessed by qRT-PCR, and the results were consistent with the findings obtained from the microarray (Fig. 2B). Of these genes, we focused on CXCL14 in the following analyses because it has not yet been reported in the context of bone metastasis.

Effects of CXCL14 silencing on bone metastases in HARA-B4inoculated nude mice. To characterize the role of CXCL14 in bone metastasis, HARA/siNC (negative control siRNA-transfected HARA), HARA-B4/siNC, and HARA-B4/siCXCL14 (siRNA that targets CXCL14-transfected HARA-B4) cells 
Table II. Effects of CXCL14 silencing on bone and adrenal metastases in HARA-B4-inoculated mice.

\section{HARA/siNC HARA-B4/siCXCL14}

Number of bone

metastases

$$
4 \text { weeks }
$$

Autopsy

$$
6.3 \pm 3.7
$$

$2.6 \pm 1.1^{\text {a }}$

$$
35.1 \pm 15.6
$$

$$
24.1 \pm 13.2
$$

Incidence of adrenal

metastases

Autopsy

$7 / 0$

$8 / 8^{\mathrm{b}}$

The number of bone metastases in the hind legs was counted by radiography at 4 weeks and 7-8 weeks (at autopsy) after inoculation of the cells. The incidence of visceral metastasis (adrenal metastasis) was also examined at autopsy. Mean $\pm \mathrm{SD}(\mathrm{n}=7-8) .{ }^{\mathrm{a}} \mathrm{p}<0.05 ;{ }^{b} \mathrm{p}<0.01$ compared to HARA-B4/siNC-inoculated mice.

were inoculated into the hearts of nude mice. Accordingly, $6.3 \pm 3.7$ and $2.6 \pm 1.1$ metastatic colonies were detected in HARA-B4/siNC- and HARA-B4/siCXCL14-inoculated mice 4 weeks after inoculation, respectively (Fig. 3 and Table II). This indicates that the silencing of CXCL14 significantly reduces bone metastases in HARA-B4-inoculated nude mice. The number of bone metastases and the incidence of adrenal metastasis were also examined at autopsy, which was performed at 7-8 weeks post-inoculation. The number of bone metastases did not significantly differ between HARA-B4/ siNC and HARA-B4/siCXCL14-inoculated nude mice at autopsy, although it tended to be lower in the HARA-B4/ siCXCL14-inoculated mice (Table II). Interestingly, none of the HARA-B4/siNC-inoculated mice exhibited adrenal gland metastases, whereas all the HARA-B4/siCXCL14-inoculated mice exhibited metastases to this organ (Table II).

Effects of CXCL14-silencing on anchorage-independent proliferation of HARA-B4. Anchorage-independent proliferation is one of the hallmarks of malignant tumor cells. Therefore, we assessed the anchorage-independent proliferation of HARA/siNC and HARA-B4/siNC using soft agar colony formation assays. One week after plating, both HARA/ siNC and HARA-B4/siNC cells formed colonies in soft agar. However, the diameters of the HARA-B4/siNC colonies were 2-3 times larger than those of the HARA/siNC colonies (Fig. 4A). Next, we examined the effect of siCXCL14-silencing in HARA-B4 on anchorage-independent proliferation activity. The colony formation activity of HARA-B4 was suppressed by siRNA-induced CXCL14 silencing (Fig. 4A). The total volume of HARA-B4/siNC colonies was more than 5 times larger than that of HARA/siNC colonies as quantified by the MTT assay, and the total volume was decreased more than 10-fold by CXCL14 silencing (Fig. 4B). The efficiency of CXCL14 silencing in HARA-B4 was checked by qRT-PCR $48 \mathrm{~h}$ after transfection (Fig. 4C).
A

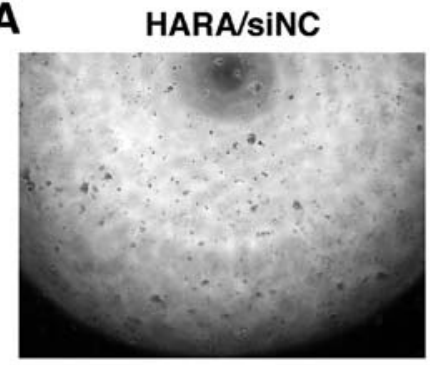

B

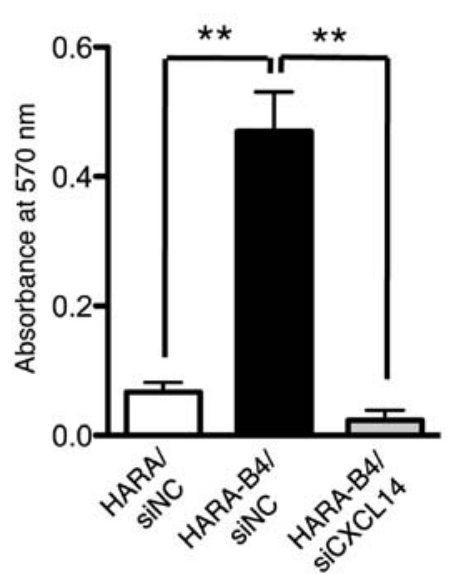

HARA-B4/siNC

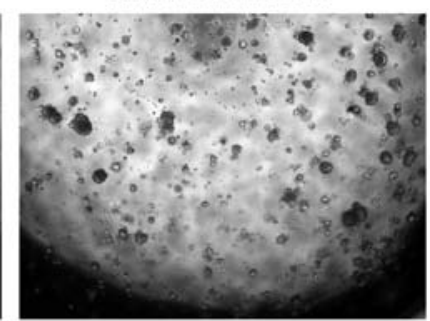

C

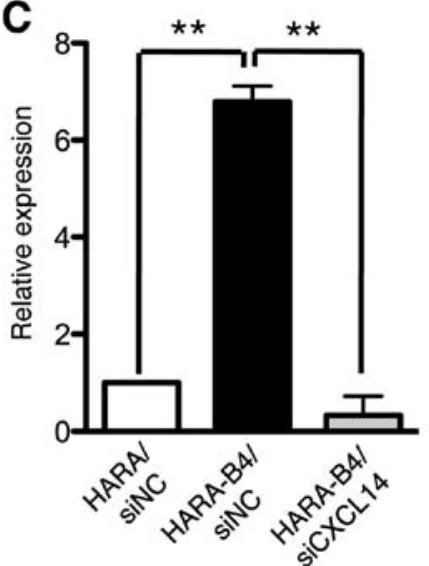

Figure 4. Effect of CXCL14 silencing on the anchorage-independent proliferation of HARA-B4. (A) Anchorage-independent proliferation measured using colony formation assays. Cells $\left(5 \times 10^{3}\right.$ cells/well) were cultured for 7 days in medium containing $1 \mathrm{X}$ CytoSelect agar matrix solution. The cells were examined microscopically 1 week after inoculation. (B) Colony volumes were calculated according to absorbance values at $570 \mathrm{~nm}$ using the MTT assay. Columns, mean (n=3); bars, SD. ${ }^{* *} \mathrm{p}<0.01$. (C) The $C X C L 14$ silencing efficiency in HARA-B4 was checked by qRT-PCR $48 \mathrm{~h}$ after transfection. Columns, mean (n=3); bars, $\mathrm{SD} .{ }^{* *} \mathrm{p}<0.01$. 
A
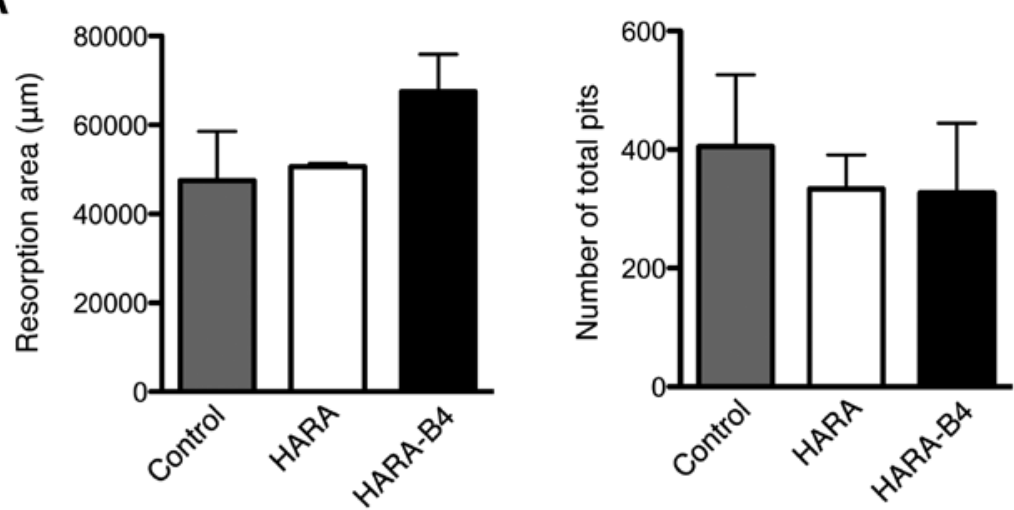

B
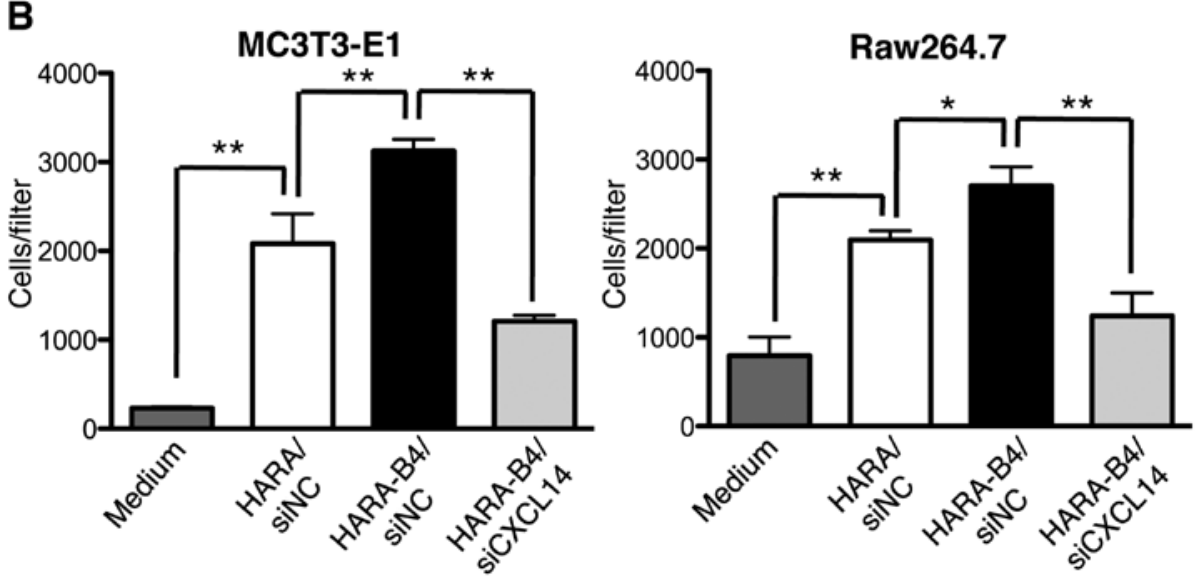

Figure 5. Interaction between HARA-B4 and the bone microenvironment. (A) Resorbing activity of osteoclasts. Mature osteoclasts were cocultured with HARA or HARA-B4 cells on Osteologic discs. The total area of resorption pits (left) and the total number of resorption pits (right) per well were measured using an image analysis system. Cancer cells were omitted in controls. Columns, mean $(n=3)$; bars, SD. (B) Transwell cell migration assay. Transwell cell migration of MC3T3-E1 and RAW264.7 cells was evaluated using a 24-well chemotaxis chamber. HARA and HARA-B4 (2.5x10 ${ }^{4}$ cells/0.8 ml) transfected with various RNAi duplexes as indicated in the figure were cultured in the lower chamber for $16 \mathrm{~h}$. Cell suspensions of MC $3 \mathrm{~T} 3-\mathrm{E} 1\left(1.25 \times 10^{4} \mathrm{cells} / 0.5 \mathrm{ml}\right) \mathrm{and}$ RAW264.7 $\left(1.25 \times 10^{5}\right.$ cells $\left./ 0.5 \mathrm{ml}\right)$ were then added to the upper chamber and incubated for 4.5 and $6 \mathrm{~h}$, respectively. Non-migrated cells were wiped off, the filter was stained with Diff-Quick, and the number of remaining cells was counted under a microscope. Columns, mean $(n=3) ;$ bars, $S D$. ${ }^{*} p<0.05 ;{ }^{* *} p<0.01$.

Interaction between cancer cells and bone marrow cells in the microenvironment of bone metastasis. To investigate the interaction between lung cancer cells and bone marrow cells in the bone microenvironment, the effects of HARA and HARA-B4 on the resorbing activity of mature osteoclasts was examined by pit formation. There were no differences in resorption areas or the number of total pits between HARA and HARA-B4 (Fig. 5A). Next, the chemotactic interactions between cancer cells and bone marrow cells were evaluated using the transwell migration chamber. When we put the murine pre-osteoblast cell line MC3T3-E1 and the pre-osteoclast/macrophage cell line RAW264.7 in the upper chamber with HARA/siNC and HARA-B4/siNC cells in the lower chamber as stimulants, both MC3T3-E1 and RAW264.7 migrated faster toward the cultured HARA-B4/siNC cells than toward the HARA/ siNC cells (Fig. 5B). We then silenced CXCL14 expression in HARA-B4 cells to evaluate the effect of CXCL14 on the migration of MC3T3-E1 and RAW264.7. Consequently, a decrease in the migration of both MC3T3-E1 and RAW264.7 was observed (Fig. 5B).

Histological examination of bone metastasis. Hematoxylin and eosin staining showed that the bone marrow cavity included infiltrated tumor cells in the bone metastatic specimens of the mice inoculated with HARA/siNC and HARA-B4/siNC (Fig. 6A and C). Expression of CXCL14 was confirmed immunohistologically in the tumor cells that infiltrated bone marrow; however, its expression patterns were patchy for HARA/siNC (Fig. 6B) and abundant and diffuse for HARA-B4/siNC (Fig. 6D). Expression of CXCL14 was also noted in stromal cells in the bone metastatic microenvironment (Fig. 6B and D). In contrast, in soft tissue metastases of mice inoculated with HARA-B4/siCXCL14, expression of CXCL14 was not observed in the infiltrated tumor cells but rather in the stromal cells (Fig. 6E and F). In the clinical specimens of lung cancer-induced bone metastasis, expression of CXCL14 was observed in the tumor cells that infiltrated the bone marrow as well as in the bone stromal cells in all specimens examined (Fig. 6G and $\mathrm{H}$ ).

\section{Discussion}

The incidence of bone metastasis has been increasing in all cancers, given the prolonged survival rate enabled by recent advances in cancer treatment. Therefore, it is important to understand how these patients should be managed in order to 

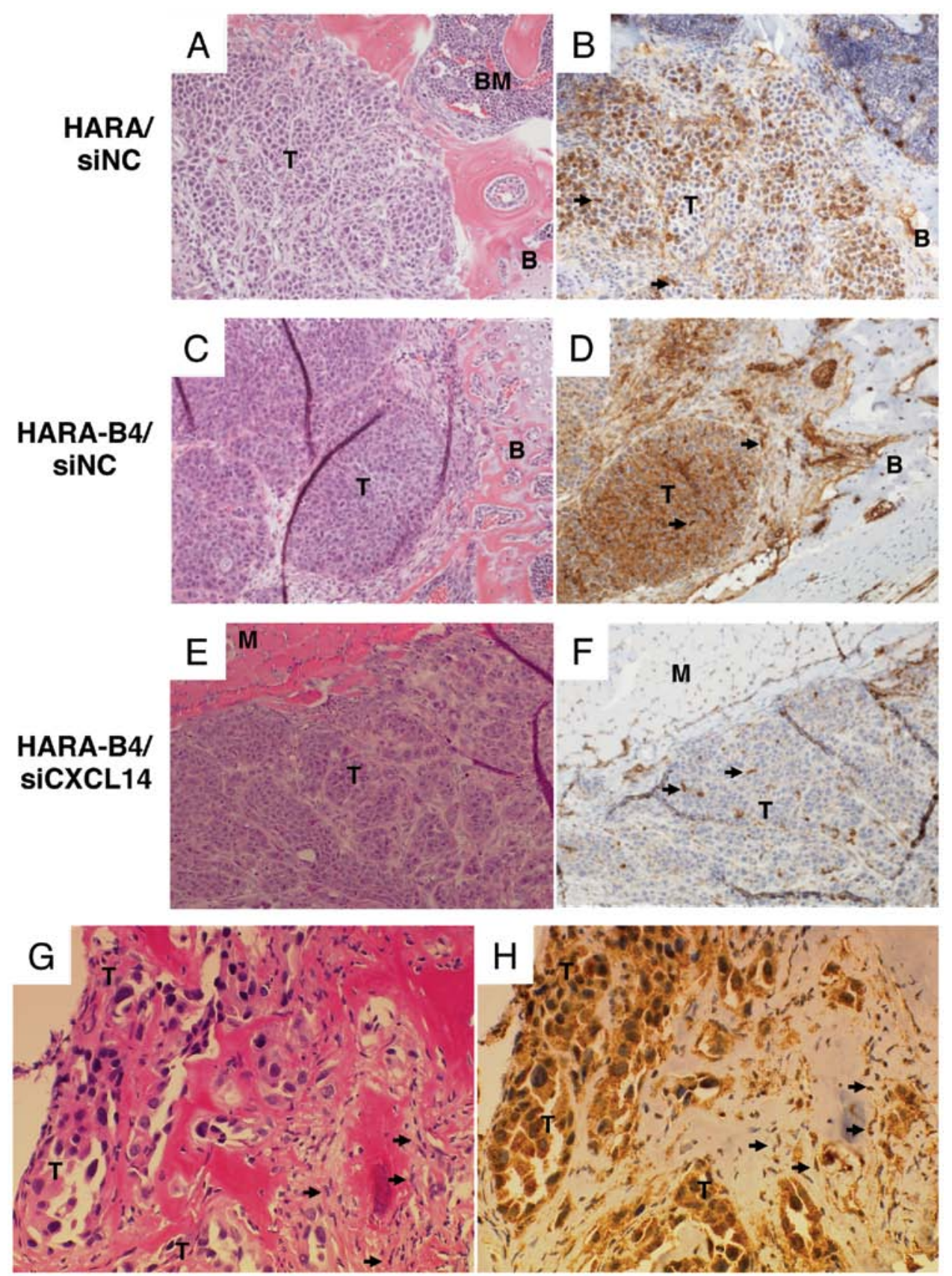

Figure 6. Histological views and expression of CXCL14 in lung cancer-induced bone metastases and/or soft tissue metastases. (A, C, E and G) Hematoxylin and eosin staining, original magnification (OM) x200. (B, D, F and H) Immunohistochemical staining of CXCL14, OM x200. Specimens of bone metastases were isolated from nude mice injected with HARA/siNC and HARA-B4/siNC at 2 weeks after inoculation. Specimens of soft tissue metastases were isolated from nude mice injected with HARA-B4/siCXCL14 because bone metastasis was not observed in these mice. Bone marrow was replaced with infiltrated tumor cells in mice injected with HARA/siNC (A) and HARA-B4/siNC (C). Expression of CXCL14 was observed in approximately half of the HARA/siNC cells (B), whereas it was observed in most HARA-B4/siNC cells (D). Expression of CXCL14 was also noted in tumor stromal cells and bone marrow stromal cells in these specimens (B and D). In the soft tissue metastases of mice injected with HARA-B4/siCXCL14, no CXCL14 expression was noted in tumor cells, and CXCL14 expression was observed only in tumor stromal cells (arrow) (E and F). (G) Lung adenocarcinoma metastasized to bone in clinical specimens (hematoxylin and eosin, OM, x200). (H) Expression of CXCL14 was observed in both tumor cells and stromal cells (arrow) in bone metastases (OM, x200). $\mathrm{T}$, tumor cells; $\mathrm{B}$, bone; $\mathrm{BM}$, bone marrow; $\mathrm{M}$, muscle; arrows, stromal cells.

maintain their QOL. The molecular mechanism responsible for bone metastasis formation has been elucidated over the past 20 years, and osteoclasts have been shown to play pivotal roles during this process. Therefore, bisphosphonates (16) and denosumab (17), which target osteoclasts, have been developed for the treatment of bone metastasis. Although these drugs are widely used in clinical practice, their clinical benefit is limited. Therefore, the development of a new drug that further improves the QOL of patients with bone metastasis is necessary.

To identify novel factors related to bone metastasis, we established a bone-seeking subclone (HARA-B4) from the parental cell line (HARA) established from human squamous cell lung cancer. We then compared the gene expression patterns between HARA and HARA-B4. From these analyses, PTHLH, MMP2, ADM and CXCL14 were identified as factors 
that are related to bone metastasis formation based on their increased gene expression in HARA-B4 and previous reports examining cancer proliferation and metastasis. Among these factors, PTHLH and MMP2 have been previously reported to contribute to bone metastasis through the differentiation and activation of osteoclasts and/or the promotion of angiogenesis $(8,20)$. ADM was also reported to be involved in the promotion of angiogenesis (21). Although CXCL14 was identified as a member of the CXC chemokine family in $1999(22,23)$, a specific receptor for CXCL14 has not yet been uncovered. CXCL14 has been described in the context of cancer cell proliferation and metastasis; however, its relationship to bone metastasis has not been elucidated (24-27). Recent studies indicate that the interaction between a chemokine (CXCL12/ SDF-1 $\alpha$ ) and its receptor (CXCR4) plays an important role in the establishment of bone metastasis $(28,29)$. Furthermore, PTHLH induces chemokine (CCL2) production from osteoblasts and other bone stromal cells, which in turn stimulates VEGF expression in tumor cells and enhances angiogenesis (30). Accordingly, chemokines and/or chemokine receptors have been shown to play important roles in bone metastasis formation. Thus, we chose to focus on CXCL14 and analyze its function in relation to bone metastasis. Indeed, mice inoculated with HARA-B4 in which CXCL14 expression was silenced exhibited a reduction in bone metastases and a simultaneous increase in visceral (adrenal) metastases, suggesting a possible correlation between the expression of CXCL14 and cancer cell tropism to the bone. HARA-B4 also exhibited an increase in anchorage-independent growth ability, in the soft agar colony formation assay, which is a hallmark of malignant cells that form tumors in vivo and is thought to be associated with highly metastatic cancer cells $(31,32)$. This anchorage-independent growth ability of HARA-B4 was decreased by CXCL14 silencing, suggesting a possible correlation between the expression of CXCL14 and the tumorigenicity of HARA-B4. Consequently, the HARA-B4 established in the present study had acquired both tropism to the bone and tumorigenicity in a bone microenvironment through increased expression of CXCL14, which resulted in the acquisition and enhancement of bone metastatic potential.

Current evidence indicates that osteoclasts play a crucial role in the development of bone metastasis (3). Bone resorption induced by activated osteoclasts creates spaces for the proliferation of metastatic cancer cells, and concurrently, growth factors released from the bone matrix create an attractive microenvironment for the growth of metastatic cancer cells (4). In the present study, the effects of HARA and HARA-B4 on the resorbing activity of mature osteoclasts were examined by pit formation; however, there were no differences between HARA and HARA-B4. The interaction between bone marrow cells and metastatic cancer cells is important for the formation of a bone metastatic niche (5); we therefore evaluated the chemotaxis of osteoblast-like cells (MC3T3-E1) and osteoclast-like cells (RAW264.7) toward HARA or HARA-B4 in vitro. The chemotaxis of both MC3T3-E1 and RAW264.7 toward HARA-B4 was significantly increased compared with that toward HARA, and furthermore, CXCL14 silencing in HARA-B4 suppressed the chemotaxis of these bone marrow cells toward HARA-B4. It is interesting to note that CXCL14 regulates the chemotaxis of bone marrow cells (osteoclasts, osteoblasts, etc.) toward metastatic cancer cells. Indeed, osteoclastogenic factors such as PTHLH and MMP2 are highly expressed in HARA-B4; therefore, it is readily conceivable that when bone marrow cells are accumulated around metastatic cancer cells, differentiation and activation of osteoclasts is facilitated by the interactions between these cells, and increased bone resorption aids the formation of metastatic cancer cell colonies. To determine whether the stimulation of chemotaxis by HARA-B4 was due to a direct effect of CXCL14, we examined the effect of recombinant CXCL14 (100 nM) on the chemotaxis of RAW267.4 and MC3T3-E1 toward HARA or HARA-B4. However, we did not observe any differences in chemotaxis (data not shown). Regarding the role of CXCL14 as a chemoattractant, a recent report indicated that CXCL14-producing mouse fibroblasts, but not recombinant CXCL14, enhanced the in vitro proliferation and migration of prostate cancer cells by undefined factors (26). A similar mechanism might be involved in the chemotaxis of bone marrow cells toward CXCL14-overproducing cancer cells observed in the present study. Further experiments will be required to answer this question.

Finally, we performed immunohistochemical staining to confirm the expression of CXCL14 in the specimens of bone metastases isolated from nude mice. Expression of CXCL14 was observed in both HARA and HARA-B4 cells that infiltrated bone marrow; however, its expression was patchy in HARA cells and abundant and diffuse in HARA-B4 cells. These results may be explained by differences in CXCL14 expression in the whole population: the HARA population is heterogeneous in terms of CXCL14 expression, whereas the HARA-B4 population consists of a homogeneous population of CXCL14-overexpressing cells enriched through in vivo selection. Similarly, in 7 clinical specimens of lung cancerinduced bone metastasis, strong expression of CXCL14 was observed in tumor cells infiltrated in bone marrow. It is interesting to note that CXCL14 expression was observed in the bone marrow stromal cells in addition to the cancer cells that infiltrated bone marrow in the mouse xenograft model as well as in the clinical specimens. These results suggest that bone marrow stromal cells, in cooperation with metastatic cancer cells, contribute to bone metastasis through a mechanism mediated by CXCL14.

In conclusion, CXCL14 was able to promote bone metastasis through enhancement of cancer cell tropism to the bone and/or recruitment of bone marrow cells (osteoclasts, osteoblasts, and others) around the metastatic cancer cells. This molecular mechanism is different from those reported previously regarding osteoclastogenic factors, which stimulate differentiation and activation of osteoclasts (3-5). Although CXCL14 plays an important role during the establishment of bone metastasis, it remains unclear whether the effects of CXCL14 are mediated directly or indirectly. Thus, further studies will be required to develop molecular therapy targeting CXCL14.

\section{Acknowledgements}

This study was supported in part by the Grant-in-Aid for Cancer Research (project no. 00054) from the Ministry of Health, Labour, and Welfare of Japan (to H. Iguchi) and Grant-in-Aid 
for Scientific Research (project no. 25462356) from Japan Society for the Promotion of Science (to S. Takiguchi).

\section{References}

1. Mundy GR: Metastasis to bone: causes, consequences and therapeutic opportunities. Nat Rev Cancer 2: 584-593, 2002.

2. Chambers AF, Groom AC and MacDonald IC: Dissemination and growth of cancer cells in metastatic sites. Nat Rev Cancer 2 : 563-572, 2002

3. Roodman GD: Mechanisms of bone metastasis. N Engl J Med 350: 1655-1664, 2004

4. Weilbaecher KN, Guise TA and McCauley LK: Cancer to bone: a fatal attraction. Nat Rev Cancer 11: 411-425, 2011.

5. Ell B and Kang Y: SnapShot: bone metastasis. Cell 151: 690-690. e1, 2012.

6. Iguchi H, Tanaka S, Ozawa Y, et al: An experimental model of bone metastasis by human lung cancer cells: the role of parathyroid hormone-related protein in bone metastasis. Cancer Res 56 4040-4043, 1996

7. Guise TA, Yin JJ, Taylor SD, et al: Evidence for a causal role of parathyroid hormone-related protein in the pathogenesis of human breast cancer-mediated osteolysis. J Clin Invest 98 : 1544-1549, 1996.

8. Lynch CC: Matrix metalloproteinases as master regulators of the vicious cycle of bone metastasis. Bone 48: 44-53, 2011.

9. Ara T and Declerck YA: Interleukin-6 in bone metastasis and cancer progression. Eur J Cancer 46: 1223-1231, 2010.

10. Croucher PI, Shipman CM, Lippitt J, et al: Osteoprotegerin inhibits the development of osteolytic bone disease in multiple myeloma. Blood 98: 3534-3540, 2001.

11. Abe M, Hiura K, Wilde J, et al: Role for macrophage inflammatory protein (MIP)-1alpha and MIP-1beta in the development of osteolytic lesions in multiple myeloma. Blood 100: 2195-2202, 2002.

12. Bendre MS, Gaddy-Kurten D, Mon-Foote T, Akel NS, Skinner RA, Nicholas RW and Suva LJ: Expression of interleukin 8 and not parathyroid hormone-related protein by human breast cancer cells correlates with bone metastasis in vivo. Cancer Res 62: 5571-5579, 2002.

13. Bendre MS, Margulies AG, Walser B, et al: Tumor-derived interleukin-8 stimulates osteolysis independent of the receptor activator of nuclear factor-kappaB ligand pathway. Cancer Res 65: 11001-11009, 2005.

14. Mancino AT, Klimberg VS, Yamamoto M, Manolagas SC and Abe E: Breast cancer increases osteoclastogenesis by secreting M-CSF and upregulating RANKL in stromal cells. J Surg Res 100: 18-24, 2001.

15. Park BK, Zhang H, Zeng Q, et al: NF-kappaB in breast cancer cells promotes osteolytic bone metastasis by inducing osteoclastogenesis via GM-CSF. Nat Med 13: 62-69, 2007.

16. Coleman R, Cook R, Hirsh V, Major P and Lipton A: Zoledronic acid use in cancer patients: more than just supportive care? Cancer 117: 11-23, 2011.

17. Lacey DL, Boyle WJ, Simonet WS, et al: Bench to bedside: elucidation of the OPG-RANK-RANKL pathway and the development of denosumab. Nat Rev Drug Discov 11: 401-419, 2012.
18. Kukita A, Kukita T, Shin JH and Kohashi O: Induction of mononuclear precursor cells with osteoclastic phenotypes in a rat bone marrow culture system depleted of stromal cells. Biochem Biophys Res Commun 196: 1383-1389, 1993.

19. Kukita A, Kukita T, Hata K, Kurisu K and Kohashi O: Heattreated osteoblastic cell (ROS17/2.8)-conditioned medium induces the formation of osteoclast-like cells. Bone Miner 23: 113-127, 1993.

20. Inoue D and Matsumoto T: Parathyroid hormone-related peptide and bone: pathological and physiological aspects. Biomed Pharmacother 54 (Suppl 1): S32-S41, 2000

21. Nikitenko LL, Fox SB, Kehoe S, Rees MC and Bicknell R: Adrenomedullin and tumour angiogenesis. Br J Cancer 94: 1-7, 2006.

22. Hromas R, Broxmeyer HE, Kim C, Nakshatri $H$, Christopherson K II, Azam M and Hou YH: Cloning of BRAK, a novel divergent CXC chemokine preferentially expressed in normal versus malignant cells. Biochem Biophys Res Commun 255: 703-706, 1999.

23. Cao X, Zhang W, Wan T, et al: Molecular cloning and characterization of a novel CXC chemokine macrophage inflammatory protein-2 gamma chemoattractant for human neutrophils and dendritic cells. J Immunol 165: 2588-2595, 2000.

24. Allinen M, Beroukhim R, Cai L, et al: Molecular characterization of the tumor microenvironment in breast cancer. Cancer Cell 6: 17-32, 2004

25. Shellenberger TD, Wang M, Gujrati M, et al: BRAK/CXCL14 is a potent inhibitor of angiogenesis and a chemotactic factor for immature dendritic cells. Cancer Res 64: 8262-8270, 2004.

26. Augsten M, Hagglof C, Olsson E, et al: CXCL14 is an autocrine growth factor for fibroblasts and acts as a multi-modal stimulator of prostate tumor growth. Proc Natl Acad Sci USA 106: 3414-3419, 2009.

27. Pelicano H, Lu W, Zhou Y, Zhang W, Chen Z, Hu Y and Huang P: Mitochondrial dysfunction and reactive oxygen species imbalance promote breast cancer cell motility through a CXCL14-mediated mechanism. Cancer Res 69: 2375-2383, 2009.

28. Muller A, Homey B, Soto H, et al: Involvement of chemokine receptors in breast cancer metastasis. Nature 410: 50-56, 2001.

29. Taichman RS, Cooper C, Keller ET, Pienta KJ, Taichman NS and McCauley LK: Use of the stromal cell-derived factor-1/CXCR4 pathway in prostate cancer metastasis to bone. Cancer Res 62: 1832-1837, 2002.

30. Li X, Loberg R, Liao J, Ying C, Snyder LA, Pienta KJ and McCauley LK: A destructive cascade mediated by CCL2 facilitates prostate cancer growth in bone. Cancer Res 69: 1685-1692, 2009.

31. Chiarugi P and Giannoni E: Anoikis: a necessary death program for anchorage-dependent cells. Biochem Pharmacol 76: 1352-1364, 2008.

32. Taddei ML, Giannoni E, Fiaschi T and Chiarugi P: Anoikis: an emerging hallmark in health and diseases. J Pathol 226: 380-393, 2012. 